\title{
The appearance of oestrone sulphate in the peripheral plasma of the pig early in pregnancy
}

\author{
H. A. Robertson*, G. J. King† and G. W. Dyck $\$$ \\ *Animal Research Institute, Ottawa, Ontario K1 A 0C6, \\ $\uparrow$ Department of Animal and Poultry Science, University of Guelph, Guelph, Ontario NIG 2Wl, and \\ $\ddagger$ Brandon Research Station, Research Branch, Agriculture Canada, Brandon, Manitoba R7A 5Z7, \\ Canada
}

\begin{abstract}
Summary. Detectable concentrations of oestrone sulphate were present in $50 \%$ of the plasma samples collected from pregnant animals by Day 17. No oestrone sulphate was detected in plasma from cyclic nonpregnant pigs.
\end{abstract}

\section{Introduction}

Robertson \& King (1974) found oestrone sulphate in the maternal plasma of a pregnant pig as early as Day 16. The purpose of the present study was to confirm and extend this finding.

\section{Materials and Methods}

Fifty (50) blood samples were collected from a total of 10 gilts between Days 13 and 29 of pregnancy (the first day of oestrus was considered to be Day 0). Regular sampling was carried out every 2nd or 3rd day on most of the animals. In addition, 13 samples were collected from three nonpregnant sows between Days 8 and 20 of the oestrous cycle.

Blood was collected into heparinized tubes by ear vein puncture, immediately chilled in iced water, centrifuged and the plasma stored at $-20^{\circ} \mathrm{C}$ in $2.5 \mathrm{ml}$ aliquots. Oestrone sulphate was determined by the method of Loriaux, Ruder \& Lipsett (1971) as modified by Robertson \& King (1974) and Tsang (1974). All estimations were carried out in duplicate on $1 \mathrm{ml}$ plasma, were individually corrected for recovery and have been expressed as oestrone equivalents. To correct for the actual gravimetric amounts of oestrone sulphate, the values should be multiplied by 1.4 . The sensitivity of the method was estimated to be $40 \mathrm{pg}$ when half the sample was taken to estimate the recovery by using an internal $\left[{ }^{3} \mathrm{H}\right]$ oestrone sulphate standard and the mean overall recovery was $65 \%$.

\section{Results and Discussion}

When the logarithm of the concentration of plasma oestrone sulphate was plotted against time the resulting curve was linear over the period from Day 20 to Day 26 (Text-fig. 1). This linear portion included the values for 30 samples collected from 8 animals and all were greater than the sensitivity of the assay method $(40 \mathrm{pg} / \mathrm{ml})$. Regression analysis of the results between Days 20 and 26 yielded the equation, $\log y=0.201 x-1.941(r=0.91)$. The values obtained for Day 17 could not be used because detectable levels were present in only some of the samples. From Day 26 to Day 29 the line flattened out. If it is assumed that the linear trend is present before Day 20 and the line is extrapolated, the minimum detectable level of oestrone sulphate $(40 \mathrm{pg} / \mathrm{ml})$ would be present by Day $17 \cdot 5$. Of the 8 samples collected on Day 17, 4 had oestrone sulphate concentrations above the sensitivity limit of the assay, and these ranged from 41 to $254 \mathrm{pg} / \mathrm{ml}$. Since the plasma concentration in some animals was already elevated by Day 17 it is evident that oestrone sulphate will be detectable in some sows at an earlier stage of pregnancy. No unconjugated oestrogens were detected in any of the samples. In the 13 samples from the 3 nonpregnant sows the plasma oestrone sulphate concentrations were all estimated to be lower than $20 \mathrm{pg} / \mathrm{ml}$. 


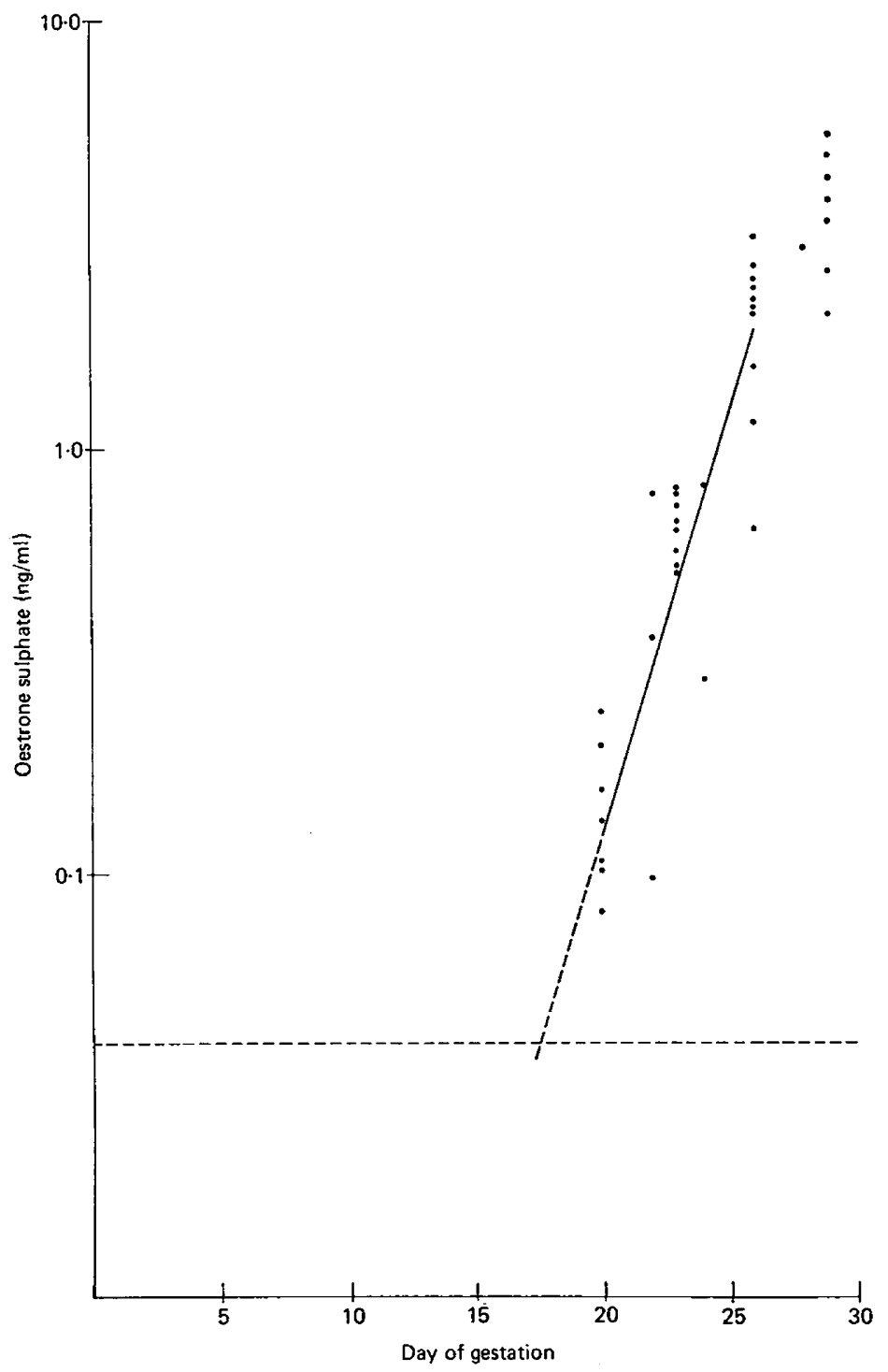

Text-fig. 1. Oestrone sulphate concentrations (as oestrone quivalent) in the plasma of the pig during early pregnancy. The horizontal dashed line indicates the minimum level detectable $(40 \mathrm{pg} / \mathrm{ml})$.

These results and those of Robertson \& King (1974) suggest that the presence of oestrone sulphate in the maternal plasma of the pig after Day 20 of pregnancy can be considered to be indicative of pregnancy in this species, and measurement of oestrone sulphate can therefore be used as a pregnancy test.

This paper is Contribution No. 719 of the Animal Research Institute.

\section{References}

LoRIAUX, D.L., Ruder, H.J. \& LipSeTt, M.B. (1971) The measurement of oestrone sulphate in plasma. Steroids 18, 463-472.

Robertson, H.A. \& KING, G.J. (1974) Plasma concentrations of progesterone, oestrone, oestradiol-17 $\beta$ and of oestrone sulphate in the pig at implantation, during pregnancy and at parturition. J. Reprod. Fert. 40, 133-141.

Tsang, C.P.W. (1974) Changes in plasma levels of estrone sulphate and estrone in the pregnant ewe around parturition. Steroids 23, 855-868.

Received 15 August 1977 